Bull. Austral. Math. Soc.

$22 \mathrm{E} 30,35 \mathrm{~B} 40$

VOL. 67 (2003) [393-406]

\title{
DERIVATIVES OF KERNELS ASSOCIATED TO COMPLEX SUBELLIPTIC OPERATORS
}

\author{
A.F.M. TER ELST
}

\begin{abstract}
We prove large time Gaussian bounds for the derivatives of the semigroup kernel associated with complex, second-order, subelliptic operators on Lie groups of polynomial growth.
\end{abstract}

\section{INTRODUCTION}

It is well established that the kernel $K$ of the semigroup generated by a right invariant sublaplacian on a connected Lie group with polynomial growth satisfies global Gaussian upper bounds. (See [15, Theorem VIII.2.9], or [12, Theorem IV.4.16].) Moreover, multiple subelliptic derivatives of the kernel satisfy small time Gaussian upper bounds with each derivative contributing an extra $t^{-1 / 2}$-singularity ([7]). Saloff-Coste [13] also proved similar bounds for single subelliptic derivatives of $K$ and large $t$. Alexopoulos [2], however, gave an example of a solvable Lie group of polynomial growth for which certain second-order subelliptic derivatives of the kernel have a $t^{-1 / 2}$, and not a $t^{-1}$, asymptotic behaviour. Nevertheless the higher order derivatives of the kernel do have global Gaussian bounds with an additional $t^{-1 / 2}$-singularity for each derivative if the group is nilpotent $([15,10])$. Moreover, for compact groups spectral arguments show that one has an exponential decrease for large $t$. The situation was clarified by the proof ([11]) that a $t^{-1}$-asymptotic behaviour is valid for the second-order subelliptic derivatives of the kernel if, and only if, the group is the (local) direct product of a compact group and a nilpotent group, and then the higher-order derivatives have a similar canonical behaviour. Despite this natural limitation Alexopoulos [1, Corollary of Theorem 7.7], showed that a general first-order derivative and some second-order derivatives of the kernel do satisfy the canonical large $t$ Gaussian bounds. The situation then developed with a recent paper

Received 12th August, 2002

On leave from Department of Mathematics and Computer Science, Eindhoven University of Technology, P.0. Box 513, $5600 \mathrm{MB}$ Eindhoven, The Netherlands. The author wishes to thank Nick Dungey and Derek Robinson for many valuable discussions. The author is particularly indebted to Nick Dungey for explaining his results prior to publication. This paper has been written whilst the author was on sabbatical leave visiting the Centre for Mathematics and its Applications at the ANU. He wishes to thank the CMA for its hospitality and the support from a grant of the Australian Research Council.

Copyright Clearance Centre, Inc. Serial-fee code: 0004-9727/03 \$A2.00+0.00. 
of [3] which proved canonical large time Gaussian bounds for multiple derivatives in the directions of the nilradical of $G$. Dungey's results were derived by combination of the Gaussian bounds on the kernel and $L_{2}$-bounds on its multiple derivatives. Since Gaussian bounds are now known for the semigroup kernels associated with complex second-order subelliptic operators ([9]) Dungey's results extend to this case. Dungey also established a $t^{-1 / 2}$-decay for $L_{2}$-bounds for any right invariant derivative of the semigroup ([4]) using an elaborate transference argument. In this paper we give a simpler proof of Dungey's results for multiple derivatives in the directions of the nilradical, based on interpolation arguments, almost as a corollary prove a $t^{-1 / 2}$-decay for any right invariant derivative of the kernel, extend Alexopoulos' result on general derivatives to the complex setting and in addition consider higher order derivatives.

Let $G$ be a connected Lie group with polynomial growth and Lie algebra $g$. In order to describe the main theorem we need some decomposition theory, which can be found in $[1$, Sections 2 and 3]. Let $\mathfrak{q}, \mathfrak{n}$ and $\mathfrak{m}$ be the radical, nilradical and a Levi subalgebra for $\mathfrak{g}$. For all $a \in \mathfrak{g}$ let $S(a)$ and $K(a)$ denote the semisimple and nilpotent part in the Jordan decomposition of ada. Then there exists a subspace $\mathfrak{v}$ of $\mathfrak{q}$ such that $\mathfrak{q}=\mathfrak{v} \oplus \mathfrak{n}$, $[\mathfrak{m}, \mathfrak{v}]=\{0\}$ and $S(\mathfrak{v}) \mathfrak{v}=\{0\}$. Then the nilshadow of $\mathfrak{q}$ is the nilpotent Lie algebra $\mathfrak{q}_{N}=\left(\mathfrak{q},[\cdot, \cdot]_{N}\right)$ where

$$
[a, b]_{N}=[a, b]-S\left(a_{\mathfrak{v}}\right) b+S\left(b_{\mathfrak{v}}\right) a
$$

with $a_{\mathfrak{b}}, b_{\mathfrak{v}}$ the $\mathfrak{v}$-components of $a, b \in \mathfrak{q}$. If $\left\{\mathfrak{q}_{N ; k}\right\}$ denotes the lower central series of $\mathfrak{q}_{N}$, that is, $\mathfrak{q}_{N ; 1}=\mathfrak{q}_{N}$ and $\mathfrak{q}_{N ; k+1}=\left[\mathfrak{q}_{N}, \mathfrak{q}_{N ; k}\right]_{N}$ for all $k \in \mathbf{N}$, then there exist vector subspaces $\mathfrak{k}, \mathfrak{h}_{1}, \ldots, \mathfrak{h}_{\mathbf{r}}$ of $\mathfrak{q}$ such that $\mathfrak{h}_{1}=\mathfrak{v} \oplus \mathfrak{k}, \mathfrak{n}=\mathfrak{k} \oplus \mathfrak{q}_{N ; 2}$ and $\mathfrak{q}_{N ; k}=\mathfrak{q}_{N ; k+1} \oplus \mathfrak{h}_{k}$ for all $k \in\{1, \ldots, r\}$, where $r$ is the rank of $q_{N}$. Let $b_{1}, \ldots, b_{d}$ be a basis for $\mathfrak{g}$ passing through $\mathfrak{v}, \mathfrak{k}, \mathfrak{h}_{2}, \ldots, \mathfrak{h}_{r}, \mathfrak{m}$. For all $i \in\{1, \ldots, d\}$ define the weight $\omega_{i}=0$ if $b_{i} \in \mathfrak{m} \oplus \mathfrak{v}$, $\omega_{i}=1$ if $b_{i} \in \mathfrak{k}$ and $\omega_{i}=k$ if $b_{i} \in \mathfrak{h}_{k}$ with $k \geqslant 2$.

For all $a \in \mathfrak{g}$ let $d L_{G}(a)$ denote the generator of the one parameter group $t$ $\mapsto L_{G}\left(\exp _{G}(-t a)\right)$, where $L_{G}$ is the left regular representation in $G$ and $\exp _{G}$ is the exponential map. We set $B_{i}=d L_{G}\left(b_{i}\right)$. We also need multi-index notation. Set $J(d)=\bigcup_{n=0}^{\infty}\{1, \ldots, d\}^{n}$ and if $\alpha=\left(i_{1}, \ldots, i_{n}\right) \in J(d)$ set $|\alpha|=n,\|\alpha\|=\omega_{i_{1}}+\ldots+\omega_{i_{n}}$ and $B^{\alpha}=B_{i_{1}} \ldots B_{i_{n}}$.

Let $a_{1}, \ldots, a_{d^{\prime}}$ be an algebraic basis for $\mathfrak{g}$ and let $C=\left(c_{k l}\right)$ be a $d^{\prime} \times d^{\prime}$-matrix of complex coefficients. Assume $2^{-1}\left(C+C^{*}\right) \geqslant \mu I$ for some $\mu>0$. Set $H=-\sum_{i, j=1}^{d^{\prime}} c_{k l} A_{k} A_{l}$, where $A_{i}=d L_{G}\left(a_{i}\right)$. Then it follows from [7] that the closure of the subelliptic operator $H$ generates a holomorphic semigroup $S$ which has a smooth kernel $K$. If $|\cdot|^{\prime}$ is the modulus on $G$ associated to the algebraic basis $a_{1}, \ldots, a_{d^{\prime}}$ and $V^{\prime}(\rho)$ denotes the Haar measure of the ball $\left\{g \in G:|g|^{\prime}<\rho\right\}$ then it follows from [9] that $K$ satisfies good 
Gaussian bounds, that is, there exist $b, c>0$ such that

$$
\left|K_{t}\right| \leqslant c G_{b, t}
$$

for all $t>0$, where $G_{b, t}(g)=V^{\prime}(t)^{-1 / 2} e^{-b\left(|g|^{\prime}\right)^{2} t^{-1}}$.

The main result of this paper is that the derivatives of $K$ satisfy the following Gaussian upper bounds.

TheOREM 1.1. If $\alpha \in J(d)$ and $i \in\{1, \ldots, d\}$ then there exist $b, c>0$ such that

$$
\left|B^{\alpha} K_{t}\right| \leqslant c t^{-\|\alpha\| / 2} G_{b, t} \quad \text { and } \quad\left|B^{\alpha} B_{i} K_{t}\right| \leqslant c t^{-(\|\alpha\|+1) / 2} G_{b, t}
$$

for all $t \geqslant 1$.

Dungey [3] proved the Gaussian bounds $\left|B^{\alpha} K_{t}\right| \leqslant c t^{-\|\alpha\| / 2} G_{b, t}$ and $\left|B^{\alpha} A_{k} K_{t}\right|$ $\leqslant c t^{-(\|\alpha\|+1) / 2} G_{b, t}$ for multi-indices $\alpha$ in the nilradical directions and in the directions of a subalgebra $\mathfrak{s}$ of $\mathfrak{m}$ for which there exists a subalgebra $\mathfrak{g}_{0}$ of $\mathfrak{g}$ such that $\mathfrak{g}=\mathfrak{s} \oplus \mathfrak{g}_{0}$ as Lie algebras. In addition Dungey proved exponential decay $e^{-\omega t}$ for any (higher-order) derivative which contains at least one derivative in the directions of $\mathbf{s}$.

As in [3] we first prove first $L_{2}$-bounds for $B^{\alpha} S_{t}$ with derivatives in the nilpotent directions. Since our proof is shorter, we include it here.

The outline of the proof is as follows. First, we may assume that $G$ is simply connected, since the general case follows from the simply connected case by a transference as in $\left[11\right.$, p. 201]. So from now on $G$ is simply connected. Secondly, we prove $L_{2}$-bounds on the derivatives of the semigroup in the nilpotent directions. These easily transform into $L_{\infty}$-bounds on nilpotent derivatives of the kernel. Thirdly, by interpolation as in [8, Lemmas 4.2 and 4.3], one obtains Gaussian bounds on the derivatives of the kernel in the nilpotent directions. Fourthly, by the convolution property $K_{2 t}=K_{t} * K_{t}$ one can move an additional derivative in any direction to the kernel on the right. Then, by induction, the first bounds of Theorem 1.1 follow. The second bounds follow similarly once one has the correct Gaussian bounds on the derivatives $A_{i} K_{t}$ in the algebraic directions.

In Section 2 we introduce some more structure theory for Lie groups with polynomial growth and prove Theorem 1.1 for derivatives in the direction of $\mathfrak{n}$. Then in Section 3 we prove Theorem 1.1 in full.

\section{Nilpotent DERIVATIVES}

The main difficulty in the proof of Theorem 1.1 is to prove Gaussian bounds for one derivative $B_{i} K_{t}$ with $b_{i} \in \mathfrak{n}$. We shall prove these bounds in this section. First we introduce more structure theory on Lie groups with polynomial growth and in addition we introduce a scale of Lie groups and subelliptic operators.

Let $M$ and $Q$ be the connected simply connected subgroups of $G$ which have Lie algebras $\mathfrak{m}$ and $\mathfrak{q}$, respectively. Then $M \cap Q=\{e\}$ and $M$ is compact since $G$ has polynomial growth. The bounds of Theorem 1.1 are independent of the choice of $\mathfrak{h}_{1}, \ldots, \mathfrak{h}_{r}$. By 
[1, Proposition 2.3], one can, however choose $\mathfrak{h}_{1}, \ldots, \mathfrak{h}_{r}$ such that $\mathfrak{h}_{k}$ is invariant under $S(\mathfrak{b})$ and adm. By $[1$, Proposition 2.4], there exists an inner product $(\cdot, \cdot)$ on $\mathfrak{g}$ such that the subspaces $\mathfrak{v}, \mathfrak{k}, \mathfrak{h}_{2}, \ldots, \mathfrak{h}_{r}, \mathfrak{m}$ are mutually orthogonal and the operators $S(v)$ are skew-symmetric for all $v \in \mathfrak{v}$. Define the inner product $\langle\cdot, \cdot\rangle$ on $\mathfrak{g}$ by

$$
\langle a, b\rangle=\int_{M} d m(\operatorname{Ad}(m) a, \operatorname{Ad}(m) b)
$$

where $d m$ is the normalised Haar measure on $M$. Then the subspaces $\mathfrak{v}, \mathfrak{k}, \mathfrak{h}_{2}, \ldots, \mathfrak{h}_{r}, \mathfrak{m}$ are mutually orthogonal and the operators ad $a$ and $S(v)$ are skew-symmetric for all $a \in \mathfrak{m}$ and $v \in \mathfrak{v}$. We may assume that $b_{1}, \ldots, b_{d}$ is an orthonormal basis with respect to $\langle\cdot, \cdot\rangle$.

For all $u>0$ let $\gamma_{u}: \mathfrak{g} \rightarrow \mathfrak{g}$ be the linear map such that $\gamma_{u}\left(b_{i}\right)=u^{w_{i}} b_{i}$ for all $i$ $\in\{1, \ldots, d\}$, where $w_{i}=0$ if $b_{i} \in \mathfrak{m}$ and $w_{i}=k$ if $b_{i} \in \mathfrak{h}_{k}$. Next define $[\cdot, \cdot]_{u}: \mathfrak{g} \times \mathfrak{g}$ $\rightarrow \mathfrak{g}$ by

$$
[a, b]_{u}=\gamma_{u}^{-1}\left(\left[\gamma_{u}(a), \gamma_{u}(b)\right]\right) \text {. }
$$

Then $\mathfrak{g}_{u}=\left(\mathfrak{g},[\cdot, \cdot]_{u}\right)$ is a Lie algebra and $\gamma_{u}: \mathfrak{g}_{u} \rightarrow \mathfrak{g}$ a Lie algebra isomorphism. Define similarly the nilpotent Lie algebra $\mathfrak{q}_{N u}=\left(\mathfrak{q},[\cdot, \cdot]_{N u}\right)$ with

$$
[a, b]_{N u}=\gamma_{u}^{-1}\left(\left[\gamma_{u}(a), \gamma_{u}(b)\right]_{N}\right) \text {. }
$$

Then $\mathbf{q}_{N u}$ is the nilshadow of $\mathrm{g}_{u}$. If $a *_{N u} b$ denotes the Campbell-Baker-Hausdorff formula in $a$ and $b$ with respect to $[\cdot, \cdot]_{N u}$ on $\mathfrak{q}_{N u}$ then $Q_{N u}=\left(\mathfrak{q}, *_{N u}\right)$ is the connected simply connected nilpotent Lie group with Lie algebra $\mathfrak{q}_{N u}$. Set $G_{N u}=M \times Q_{N u}$. We denote by $*_{N u}$ the multiplication on $G_{N u}$ and by $g^{(-1)_{N u}}$ the inverse of $g$. Define $\tau_{u}: \mathfrak{g}_{N u} \rightarrow \mathcal{L}\left(\mathfrak{g}_{N u}\right)$ by $\tau_{\mathfrak{u}}(a) b=\left(\operatorname{ad} a_{\mathfrak{m}}+S\left(\gamma_{u}\left(a_{\mathfrak{v}}\right)\right)\right) b_{\mathfrak{q}}$, where $a_{\mathfrak{m}}$ and $a_{\mathfrak{v}}$ are the components of $a$ in $\mathfrak{m}$ and $\mathfrak{v}$ and $b_{\mathfrak{q}}$ is the component of $b$ in $\mathfrak{q}$. If $\overline{T_{u}}: \mathfrak{g}_{N u} \rightarrow \operatorname{Aut}\left(\mathfrak{g}_{N u}\right)$ is the homomorphism such that $\overline{T_{u}}\left(\exp _{G_{N u}} a\right)=e^{T_{u}(a)}$ and $T_{u}: G_{N u} \rightarrow \operatorname{Aut}\left(G_{N u}\right)$ is the Lie group homomorphism such that

$$
T\left(\exp _{G_{N u}} a\right) \exp _{G_{N u}} b=\exp _{G_{N u}}\left(\overline{T_{u}}\left(\exp _{G_{N u}} a\right) b\right)
$$

for all $a, b \in \mathfrak{g}_{N u}$ then $(g, h) \mapsto g T_{T_{u}} * h=\left(T_{u}\left(h^{(-1)_{N u}}\right) g\right) *_{N u} h$ defines a Lie group multiplication on the set $G_{N u}$ of which the Lie algebra is isomorphic to $\mathfrak{g}_{u}$ (compare [14, p. 229]). Here $\exp _{G_{N u}}$ denotes the usual exponential map on $G_{N u}$. We set $G_{u}=\left(G_{N u}, T_{u} *\right)$ and $T=T_{1}$. Then with $u=1$ the Lie group $G$ is isomorphic to $\left(G_{N u}, T^{*}\right)$ and from now on we identify $G$ with $\left(G_{N 1}, T^{*}\right)$. We also delete the $u$ in a symbol if $u=1$. As a consequence

$$
\left(d L_{G_{u}}(a) \varphi\right)(g)=\left(d L_{G_{N u}}\left(\overline{T_{u}}\left(g^{(-1)_{N u}}\right) a\right) \varphi\right)(g)
$$

for all $a \in \mathfrak{g}, g \in G_{u}$ and $\varphi \in C_{c}^{\infty}\left(G_{u}\right)$. But it follows from the skew-symmetry of adm and $S(\mathfrak{v})$ that $\bar{T}_{u}$ is a unitary representation of $G_{N u}$ on $\mathfrak{g}$ equipped with the inner product $\langle\cdot, \cdot\rangle$. 
We choose and fix a Lebesgue measure on the vector space q. Then we fix the Haar measure on $Q_{N u}$ such that $\int_{Q_{N u}} \varphi=\int_{q} \varphi \circ \exp _{Q_{N u}}$ for all $\varphi \in C_{c}\left(Q_{N u}\right)$. Then the Haar measure on $G_{N u}$ is the product measure of the normalised Haar measure on the compact group $M$ and the Haar measure on $Q_{N u}$. Finally since $\left|\operatorname{det} \overline{T_{u}}(g)\right|=1$ for all $g$ it follows that we can choose the Haar measure on $G_{u}$ such that $\int_{G_{u}} \varphi=\int_{G_{N u}} \varphi$ for all $\varphi \in C_{c}\left(G_{u}\right)$. Note that this fixes the Haar measure on $G=G_{1}$.

If $a_{1}, \ldots, a_{d^{\prime}}$ is the algebraic basis of $\mathfrak{g}$ then $u \gamma_{u}^{-1}\left(a_{1}\right), \ldots, u \gamma_{u}^{-1}\left(a_{d^{\prime}}\right)$ is an algebraic basis for $\mathfrak{g}_{u}$. Now set $A_{k}^{[u]}=d L_{G_{u}}\left(u \gamma_{u}^{-1}\left(a_{k}\right)\right)$ for all $k \in\left\{1, \ldots, d^{\prime}\right\}$. Then (2) implies that

$$
\left(A_{k}^{[u]} \varphi\right)(g)=\sum_{j=1}^{d}\left\langle\overline{T_{u}}(g) b_{j}, u \gamma_{u}^{-1}\left(a_{k}\right)\right\rangle\left(\widetilde{B}_{j}^{(u)} \varphi\right)(g)=\sum_{j=1}^{d} u^{1-w_{j}} r_{k j}^{(u)}(g)\left(\widetilde{B}_{j}^{(u)} \varphi\right)(g)
$$

where

$$
\widetilde{B}_{i}^{(u)}=d L_{G_{N u}}\left(b_{i}\right),
$$

$r_{k j}^{(u)}=r_{k j} \circ \Gamma_{u}, r_{k j}(g)=\left\langle\bar{T}(g) b_{j}, a_{k}\right\rangle$ and $\Gamma_{u}: G_{u} \rightarrow G$ is the lifting of the isomorphism $\gamma_{u}$.

Next, define the subelliptic operator $H_{\{u\}}$ on $G_{u}$ by

$$
H_{[u]}=-\sum_{k, l=1}^{d^{\prime}} c_{k l} A_{k}^{[u]} A_{l}^{[u]}
$$

If $S^{[u]}$ is the semigroup generated by $H_{[u]}$ then by subellipticity there exists a $c_{1}>0$ such that

$$
\begin{gathered}
\left\|\left(\lambda I+H_{[u]}\right)^{-1}\right\|_{2 \rightarrow 2} \leqslant \lambda^{-1} \\
\left\|\left(\lambda I+H_{[u]}\right)^{-1} A_{k}^{[u]}\right\|_{2 \rightarrow 2} \leqslant c_{1} \lambda^{-1 / 2}
\end{gathered}
$$

uniformly for all $\lambda, u>0$ and $k \in\left\{1, \ldots, d^{\prime}\right\}$.

Let $d_{0}=\operatorname{dim} \mathfrak{v}$ and $d_{q}=\operatorname{dim} \mathbf{q}$. Then $\mathfrak{n}=\left\{b_{d_{0}+1}, \ldots, b_{d_{q}}\right\}$. Moreover, set $J(\mathfrak{n})$ $=\bigcup_{n=0}^{\infty}\left\{d_{0}+1, \ldots, d_{q}\right\}^{n}$. First we prove $L_{2}$ bounds on nilpotent derivatives.

Proposition 2.1. For all $\alpha \in J(\mathfrak{n})$ there exists a $c>0$ such that

$$
\left\|B^{\alpha} S_{t}\right\|_{2 \rightarrow 2} \leqslant c t^{-\|\alpha\| / 2}
$$

for all $t \geqslant 1$.

The proof requires some preparation. For all $u>0$ consider the unitary representation $U^{(u)}$ of the Lie group $N$ in $L_{2}\left(G_{N u}\right)$ defined by $U^{(u)}(n)=L_{G_{N u}}(n)$. For $m \in \mathrm{N}_{0}$ define the space

$$
\mathcal{X}_{m}^{(u)}=\bigcap_{\alpha \in J_{m}(n)} D\left(\widetilde{B}^{(u) \alpha}\right)
$$


with norm

$$
\|\| \varphi\left\|_{m}^{(u)}=\max _{\alpha \in J_{m}(\mathfrak{n})}\right\| \widetilde{B}^{(u) \alpha} \varphi \|_{2}
$$

where $\|\cdot\|_{2}$ is the $L_{2}$-norm on $L_{2}\left(G_{N u}\right)$ and $J_{m}(\mathfrak{n})=\{\alpha \in J(\mathfrak{n}):|\alpha| \leqslant m\}$. So $\mathcal{X}_{m}^{(u)}$ is the Banach space of $m$-times differentiable elements with respect to the representation $U^{(u)}$ and the vector space basis $b_{d_{1}+1}, \ldots, b_{d_{q}}$ for $\mathfrak{n}$, with the usual norm. Define the seminorm $N_{m}^{(u)}: \mathcal{X}_{m}^{(u)} \rightarrow \mathbf{R}$ by

$$
N_{m}^{(u)}(\varphi)=\max _{\substack{\alpha \in J(\mathbf{n}) \\|\alpha|=m}}\left\|\widetilde{B}^{(u) \alpha} \varphi\right\|_{2}
$$

Next we need bounds on $N_{m}^{(u)}\left(\left(\lambda I+H_{[u]}\right)^{-1} \varphi\right)$.

LEMMA 2.2. For all $m \in \mathbf{N}$ there exist $c, \lambda_{0}>0$ such that

$$
N_{m}^{(u)}\left(\left(\lambda I+H_{[u]}\right)^{-1} \varphi\right) \leqslant c \lambda^{-1} N_{m}^{(u)}(\varphi)
$$

for all $\lambda \geqslant \lambda_{0}, u \geqslant 1$ and $\varphi \in L_{2 ; \infty}\left(G_{N u}\right)$.

PROOF: Since $\widetilde{B}_{i}^{(u)} r_{k j}^{(u)}=0$ if $b_{i} \in \mathfrak{n}$ and $\mathfrak{n}$ is an ideal it follows from (3) that there exists a $c>0$ such that

$$
\left\|\left[\widetilde{B}^{(u) \alpha}, A_{k}^{[u]}\right] \varphi\right\|_{2} \leqslant c N_{m}^{(u)}(\varphi)
$$

for all $k \in\left\{1, \ldots, d^{\prime}\right\}, u \geqslant 1, \varphi \in L_{2 ; \infty}\left(G_{N u}\right)$ and $\alpha \in J(\mathfrak{n})$ with $|\alpha|=m$. Let $u \geqslant 1$, $\lambda>0, \varphi \in L_{2 ; \infty}\left(G_{N u}\right)$ and write $\psi=\left(\lambda I+H_{[u]}\right)^{-1} \varphi$. For all $\alpha \in J(\mathfrak{n})$ with $|\alpha|=m$ one has

$$
\begin{aligned}
\left\|\widetilde{B}^{(u) \alpha} \psi\right\|_{2} \leqslant & \left\|\left(\lambda I+H_{[u]}\right)^{-1} \widetilde{B}^{(u) \alpha} \varphi\right\|_{2}+\left\|\left(\lambda I+H_{[u]}\right)^{-1}\left[\widetilde{B}^{(u) \alpha}, H_{[u]}\right] \psi\right\|_{2} \\
\leqslant & \lambda^{-1} N_{m}^{(u)}(\varphi)+\sum_{k, l=1}^{d^{\prime}}\left|c_{k l}\right|\left\|\left(\lambda I+H_{[u]}\right)^{-1}\left[\widetilde{B}^{(u) \alpha}, A_{k}^{[u]}\right] A_{l}^{[u]} \psi\right\|_{2} \\
& +\sum_{k, l=1}^{d^{\prime}}\left|c_{k l}\right|\left\|\left(\lambda I+H_{[u]}\right)^{-1} A_{k}^{[u]}\left[\widetilde{B}^{(u) \alpha}, A_{l}^{[u]}\right] \psi\right\|_{2} \\
\leqslant & \lambda^{-1} N_{m}^{(u)}(\varphi)+c \lambda^{-1} \sum_{k, l=1}^{d^{\prime}}\left|c_{k l}\right| N_{m}^{(u)}\left(A_{l}^{[u]} \psi\right)+c c_{1} \lambda^{-1 / 2} \sum_{k, l=1}^{d^{\prime}}\left|c_{k l}\right| N_{m}^{(u)}(\psi)
\end{aligned}
$$

where we used (4) and (5). So with $c_{2}=c \sum_{k, l=1}^{d^{\prime}}\left|c_{k l}\right|$ one deduces that

$$
N_{m}^{(u)}(\psi) \leqslant \lambda^{-1} N_{m}^{(u)}(\varphi)+c_{2} \lambda^{-1} \max _{k \in\left\{1, \ldots, d^{\prime}\right\}} N_{m}^{(u)}\left(A_{k}^{[u]} \psi\right)+c_{1} c_{2} \lambda^{-1 / 2} N_{m}^{(u)}(\psi)
$$

Next, if $k \in\left\{1, \ldots, d^{\prime}\right\}$ and $\alpha \in J(\mathfrak{n})$ with $|\alpha|=m$ then it follows from subellipticity 
that

$$
\begin{aligned}
\left\|\widetilde{B}^{(u) \alpha} A_{k}^{[u]} \psi\right\|_{2}^{2} & \leqslant 2\left\|A_{k}^{[u]} \widetilde{B}^{(u) \alpha} \psi\right\|_{2}^{2}+2\left\|\left[\widetilde{B}^{(u) \alpha}, A_{k}^{[u]}\right] \psi\right\|_{2}^{2} \\
& \leqslant 2 \mu^{-1} \operatorname{Re}\left(\widetilde{B}^{(u) \alpha} \psi, H_{[u]} \widetilde{B}^{(u) \alpha} \psi\right)+2 c^{2} N_{m}^{(u)}(\psi)^{2} \\
& \leqslant 2 \mu^{-1} \operatorname{Re}\left(\widetilde{B}^{(u) \alpha} \psi, \widetilde{B}^{(u) \alpha} H_{[u]} \psi\right) \\
& \quad+2 \mu^{-1} \operatorname{Re}\left(\widetilde{B}^{(u) \alpha} \psi,\left[H_{[u]}, \widetilde{B}^{(u) \alpha}\right] \psi\right)+2 c^{2} N_{m}^{(u)}(\psi)^{2} .
\end{aligned}
$$

But $H_{[u]} \psi=\varphi-\lambda \psi$. So

$$
\begin{aligned}
\operatorname{Re}\left(\widetilde{B}^{(u) \alpha} \psi, \widetilde{B}^{(u) \alpha} H_{[u]} \psi\right) & =\operatorname{Re}\left(\widetilde{B}^{(u) \alpha} \psi, \widetilde{B}^{(u) \alpha} \varphi\right)-\lambda \operatorname{Re}\left(\widetilde{B}^{(u) \alpha} \psi, \widetilde{B}^{(u) \alpha} \psi\right) \\
& \leqslant N_{m}^{(u)}(\psi) N_{m}^{(u)}(\varphi) \leqslant 2^{-1}\left(N_{m}^{(u)}(\psi)^{2}+N_{m}^{(u)}(\varphi)^{2}\right)
\end{aligned}
$$

and

$$
\begin{aligned}
& \left|\operatorname{Re}\left(\widetilde{B}^{(u) \alpha} \psi,\left[H_{[u]}, \widetilde{B}^{(u) \alpha}\right] \psi\right)\right| \\
& \quad \leqslant \sum_{i, j=1}^{d^{\prime}}\left|c_{i j}\right|\left|\left(\widetilde{B}^{(u) \alpha} \psi,\left[A_{i}^{[u]}, \widetilde{B}^{(u) \alpha}\right] A_{j}^{[u]} \psi\right)\right|+\sum_{i, j=1}^{d^{\prime}}\left|c_{i j}\right|\left|\left(\widetilde{B}^{(u) \alpha} \psi, A_{i}^{[u]}\left[A_{j}^{[u]}, \widetilde{B}^{(u) \alpha}\right] \psi\right)\right| \\
& \quad \leqslant c_{2} N_{m}^{(u)}(\psi) \max _{j \in\left\{1, \ldots, d^{\prime}\right\}} N_{m}^{(u)}\left(A_{j}^{[u]} \psi\right)+c_{2} \max _{i \in\left\{1, \ldots, d^{\prime}\right\}} N_{m}^{(u)}\left(A_{i}^{[u]} \psi\right) N_{m}^{(u)}(\psi)+c c_{2} N_{m}^{(u)}(\psi)^{2}
\end{aligned}
$$

by anti-symmetry of $A_{i}^{[u]}$ and an estimate on the commutator $\left[A_{i}^{[u]}, \widetilde{B}^{(u) \alpha}\right]$. Hence

$$
\begin{aligned}
\max _{k \in\left\{1, \ldots, d^{\prime}\right\}} N_{m}^{(u)}\left(A_{k}^{[u]} \psi\right)^{2} \leqslant \mu^{-1} N_{m}^{(u)}(\varphi)^{2}+4 c_{2} \mu^{-1} N_{m}^{(u)}(\psi) & \max _{j \in\left\{1, \ldots, d^{\prime}\right\}} N_{m}^{(u)}\left(A_{j}^{[u]} \psi\right) \\
& +2\left(\mu^{-1}+c^{2}+\mu^{-1} c c_{2}\right) N_{m}^{(u)}(\psi)^{2}
\end{aligned}
$$

Therefore

$$
\max _{k \in\left\{1, \ldots, d^{\prime}\right\}} N_{m}^{(u)}\left(A_{k}^{[u]} \psi\right) \leqslant \dot{\mu}^{-1 / 2} N_{m}^{(u)}(\varphi)+c_{3} N_{m}^{(u)}(\psi)
$$

where $c_{3}=4 c_{2} \mu^{-1}+\left(2\left(\mu^{-1}+c^{2}+\mu^{-1} c c_{2}\right)\right)^{1 / 2}$. Together with (7) it follows that

$$
N_{m}^{(u)}(\psi) \leqslant \lambda^{-1}\left(1+c_{2} \mu^{-1 / 2}\right) N_{m}^{(u)}(\varphi)+c_{2}\left(c_{3} \lambda^{-1}+c_{1} \lambda^{-1 / 2}\right) N_{m}^{(u)}(\psi) .
$$

So the lemma follows with $\lambda_{0}=1+4 c_{2}^{2}\left(c_{1}+c_{3}\right)^{2}$.

Now we are able to prove Proposition 2.1.

Proof of Proposition 2.1: Let $m \in \mathbf{N}, m \geqslant 2$. It follows from the first five steps in the proof of $\left[9\right.$, Lemma 2.2] that there exists a $\nu \in\langle 0,1\rangle$ such that $L_{2 ; 1}^{\prime}\left(G_{u}\right)$ $\subset\left(L_{2}\left(G_{N u}\right), L_{2 ; m r}^{\prime}\left(G_{N u}\right)\right)_{\gamma, \infty ; \mathrm{K}}$ and the embedding is continuous uniformly for all $u \geqslant 1$, where $L_{2 ; 1}^{\prime}\left(G_{u}\right)$ is the Sobolev space defined with respect to the group $G_{u}$ and the algebraic basis $u \gamma_{u}^{-1}\left(a_{1}\right), \ldots, u \gamma_{u}^{-1}\left(a_{d^{\prime}}\right)$ and $L_{2 ; m r}^{\prime}\left(G_{N u}\right)$ is the Sobolev space defined with respect to the group $G_{N u}$ and the algebraic basis $b_{1}, \ldots, b_{d_{1}}, b_{d_{0}+1}, \ldots, b_{d}$, where $d_{1}=\operatorname{dim} \mathfrak{h}_{1}$. Here we used the real interpolation spaces with respect to the K-method of Peetre. Obviously 
$L_{2 ; m r}^{\prime}\left(G_{N u}\right) \subset L_{2 ; m r}^{\prime(Q)}\left(G_{N u}\right)$, the Sobolev space with respect to the set $b_{1}, \ldots, b_{d_{1}}$. But $L_{2 ; m r}^{\prime(Q)}\left(G_{N 1}\right) \subseteq L_{2 ; m}^{(Q)}\left(G_{N 1}\right)$, the Sobolev space with respect to the set $b_{1}, \ldots, b_{d_{q}}$ and the embedding is continuous. Therefore, by scaling, the space $L_{2 ; m r}^{\prime(Q)}\left(G_{N_{u}}\right)$ is embedded in the space $L_{2 ; m}^{(Q)}\left(G_{N u}\right)$ and the embedding is continuous uniformly for all $u \geqslant 1$. Obviously, $L_{2 ; m}^{(Q)}\left(G_{N u}\right) \subseteq \mathcal{X}_{m}^{(u)}$ and the norm of the embedding is bounded by 1 . Combining these embeddings there exists a $c>0$ such that

$$
\|\varphi\|_{\left(\mathcal{X}_{0}^{(u)}, \mathcal{X}_{m}^{(u)}\right)_{\nu, 2, \mathrm{~K}}} \leqslant c\left(\|\varphi\|_{2}+\sum_{k=1}^{d^{\prime}}\left\|A_{k}^{(u)} \varphi\right\|_{2}\right)
$$

uniformly for all $u \geqslant 1$ and $\varphi \in L_{2 ; 1}^{\prime}\left(G_{u}\right)$. Hence by (4) and (5) there exists a $c_{0}>0$ such that

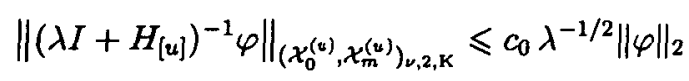

for all $u \geqslant 1, \lambda \geqslant 1$ and $\varphi \in \mathcal{X}_{0}^{(u)}$.

By Lemma 2.2 there exist $c_{1} \geqslant 1$ and $\lambda_{0}>0$ such that such that

$$
\|\|\left(\lambda I+H_{[u]}\right)^{-1} \varphi\|\|_{m} \leqslant c_{1} \lambda^{-1} \mid\|\varphi\|_{m}
$$

for all $\lambda \geqslant \lambda_{0}, u \geqslant 1$ and $\varphi \in L_{2 ; \infty}\left(G_{N u}\right)$. But $L_{2 ; \infty}\left(G_{N u}\right)$ is dense in $\mathcal{X}_{m}^{(u)}$ by an argument similar to the proof of [5, Lemma 2.4]. Hence if $\lambda=1 \vee \lambda_{0}$ then the map $\left(\lambda I+H_{[u]}\right)^{-1}$ is continuous from $\mathcal{X}_{0}^{(u)}$ into $\mathcal{X}_{0}^{(u)}$ with norm bounded by 1 and from $\mathcal{X}_{m}^{(u)}$ into $\mathcal{X}_{m}^{(u)}$ with norm bounded by $c_{1}$. Therefore, by interpolation, for all $\gamma \in\langle 0,1\rangle$ the map $\left(\lambda I+H_{[u]}\right)^{-1}$ is continuous from $\left(\mathcal{X}_{0}^{(u)}, \mathcal{X}_{m}^{(u)}\right)_{\gamma, 2, \mathrm{~K}}$ into $\left(\mathcal{X}_{0}^{(u)}, \mathcal{X}_{m}^{(u)}\right)_{\gamma, 2, \mathrm{~K}}$ with norm bounded by $c_{1}$. But by (8) the map $\left(\lambda I+H_{[u]}\right)^{-1}$ is also continuous from $\mathcal{X}_{0}^{(u)}$ into $\left(\mathcal{X}_{0}^{(u)}, \mathcal{X}_{m}^{(u)}\right)_{\nu, 2, \mathrm{~K}}$ with norm bounded by $c_{0}$. Hence, by interpolation, for all $\gamma \in\langle 0,1\rangle$ the map $\left(\lambda I+H_{[u]}\right)^{-1}$ is continuous from $\left(\mathcal{X}_{0}^{(u)}, \mathcal{X}_{m}^{(u)}\right)_{\gamma, 2, \mathrm{~K}}$ into $\left(\mathcal{X}_{0}^{(u)}, \mathcal{X}_{m}^{(u)}\right)_{\gamma+(1-\gamma) \nu, 2, \mathrm{~K}}$ with norm bounded by $c_{0}+c_{1}$. Using interpolation once more, it follows that there exists an $N \in \mathrm{N}$ such that the map $\left(\lambda I+H_{[u]}\right)^{-N}$ is continuous from $\mathcal{X}_{0}^{(u)}$ into $\left(\mathcal{X}_{0}^{(u)}, \mathcal{X}_{m}^{(u)}\right)_{1-(2 m)^{-1}, 2, \mathrm{~K}}$ with norm bounded by $\left(1+c_{0}+c_{1}\right)^{N}$. By (11) in [6] one has $\left(\mathcal{X}_{0}^{(u)}, \mathcal{X}_{m}^{(u)}\right)_{1-(2 m)^{-1}, 2, \mathrm{~K}} \subseteq \mathcal{X}_{m-1}^{(u)}$ and the embedding is continuous, uniformly for all $u>0$. Hence there exists a $c_{2}>0$ such that

$$
\left\|\widetilde{B}^{(u) \alpha} \varphi\right\|_{2} \leqslant\|\varphi\|_{m-1}^{(u)} \leqslant c_{2}\|\varphi\|_{\left(\mathcal{X}_{0}^{(u)}, \mathcal{X}_{m}^{(u)}\right)_{1-(2 m)^{-1,2, K}}}
$$

uniformly for all $u \geqslant 1, \varphi \in L_{2 ; \infty}\left(G_{N u}\right)$ and $\alpha \in J(\mathfrak{n})$ with $|\alpha|=m-1$. Then

$$
\left\|\widetilde{B}^{(u) \alpha} \varphi\right\|_{2} \leqslant c_{2}\left(1+c_{0}+c_{1}\right)^{N}\left\|\left(\lambda I+H_{[u]}\right)^{N} \varphi\right\|_{2}
$$

for all $\varphi \in L_{2 ; \infty}\left(G_{u}\right)$. In particular,

$$
\begin{aligned}
\left\|\widetilde{B}^{(u) \alpha} S_{1}^{[u]}\right\|_{2 \rightarrow 2} & \leqslant c_{2}\left(1+c_{0}+c_{1}\right)^{N} \sum_{n=0}^{N}\left(\begin{array}{c}
N \\
n
\end{array}\right) \lambda^{N-n}\left\|H_{[u]}^{n} S_{1}^{[u]}\right\|_{2 \rightarrow 2} \\
& \leqslant c_{2}\left(1+c_{0}+c_{1}\right)^{N}\left(\lambda^{N}+\sum_{n=1}^{N}\left(\begin{array}{l}
N \\
n
\end{array}\right) \lambda^{N}\left\|H_{[u]} S_{1 / n}^{[u]}\right\|_{2 \rightarrow 2}^{n}\right) .
\end{aligned}
$$


But there exists a $c_{3} \geqslant 1$ such that $\left\|H S_{t}\right\|_{2 \rightarrow 2} \leqslant c_{3} t^{-1}$ for all $t>0$. Then $\left\|H_{[u]} S_{1 / n}^{[u]}\right\|_{2 \rightarrow 2}$ $=u^{2}\left\|H S_{n^{-1} u^{2}}\right\|_{2 \rightarrow 2} \leqslant c_{3} n$ uniformly for all $u>0$. Then

$$
\left\|\widetilde{B}^{(u) \alpha} S_{1}^{[u]}\right\|_{2 \rightarrow 2} \leqslant c_{4}
$$

for all $u \geqslant 1$, where $c_{4}=c_{2} c_{3}\left(2\left(1+c_{0}+c_{1}\right) \lambda\right)^{N} N$. Finally, it follows from (2) that $B_{i}^{(u)}=\sum_{j=1}^{d}\left(\rho_{i j} \circ \Gamma_{u}\right) \widetilde{B}_{j}^{(u)}$ for all $i \in\{1, \ldots, d\}$ and $u>0$, where $\rho_{i j}(g)=\left\langle\bar{T}(g) b_{j}, b_{i}\right\rangle$. But if $b_{i} \in \mathfrak{n}$ then for a non-vanishing term in the sum one must have $b_{j} \in \mathfrak{n}$. In addition, $\widetilde{B}_{k}^{(u)} \rho_{i j}=0$ if $b_{k} \in \mathfrak{n}$ and $\rho_{i j}$ is bounded. So for all $\alpha \in J(\mathfrak{n})$ there exist bounded continuous functions $\rho_{\beta}$ such that $B^{(u) \alpha}=\sum_{\beta \in J(n) ;|\beta|=|\alpha|}\left(\rho_{\beta} \circ \Gamma_{u}\right) \widetilde{B}^{(u) \beta}$. Then for all $\alpha \in J(\mathfrak{n})$ there exists a $c_{5}>0$ such that $\left\|B^{(u) \alpha} S_{1}^{[u]}\right\|_{2 \rightarrow 2} \leqslant c_{5}$, uniformly for all $u \geqslant 1$. Then

$$
\left\|B^{\alpha} S_{t}\right\|_{2 \rightarrow 2}=t^{-\|\alpha\| / 2}\left\|B^{\left(t^{1 / 2}\right) \alpha} S_{1}^{\left(t^{1 / 2}\right]}\right\|_{2 \rightarrow 2} \leqslant c_{5} t^{-\|\alpha\| / 2}
$$

for all $t \geqslant 1$ and Proposition 2.1 follows.

COROLlary 2.3. For all $\alpha \in J(\mathfrak{n})$ there exists a $c>0$ such that

$$
\left\|B^{\alpha} K_{t}\right\|_{\infty} \leqslant c V^{\prime}(t)^{-1 / 2} t^{-\|\alpha\| / 2}
$$

for all $t \geqslant 1$.

Proof: For all $t \geqslant 1$ and $\alpha \in J(\mathfrak{n})$ one has

$$
\begin{aligned}
\left\|B^{\alpha} K_{3 t}\right\|_{\infty}=\left\|B^{\alpha} S_{3 t}\right\|_{1 \rightarrow \infty} & \leqslant\left\|B^{\alpha} S_{2 t}\right\|_{2 \rightarrow \infty}\left\|S_{t}\right\|_{1 \rightarrow 2} \\
& =\left\|B^{\alpha} S_{t}\right\|_{2 \rightarrow 2}\left\|S_{t}\right\|_{2 \rightarrow \infty}\left\|S_{t}^{*}\right\|_{2 \rightarrow \infty}=\left\|B^{\alpha} S_{t}\right\|_{2 \rightarrow 2}\left\|K_{t}\right\|_{2}\left\|K_{t}^{\dagger}\right\|_{2}
\end{aligned}
$$

where $K^{\dagger}$ is the kernel of $S^{*}$. Then the corollary follows from the bounds (6), the Gaussian bounds on $K$ and $K^{\dagger}$ and the doubling property.

The next lemma is the key in the induction step to turn $L_{\infty}$-bound on derivatives and Gaussian bounds on $K$ into Gaussian bounds on derivatives of $K$.

Lemma 2.4. Suppose $a \in \mathfrak{g}, t \geqslant 1, b, c_{0}, c_{2}, c, \delta>0, \Phi \in C^{\infty}(G)$ and suppose that

$$
|\Phi(g)| \leqslant c_{0} G_{b, t}(g)
$$

for all $g \in G$,

$$
\left\|d L(a)^{2} \Phi\right\|_{\infty} \leqslant c_{2} V^{\prime}(t)^{-1 / 2}
$$

and $|\exp u a|^{\prime} \leqslant c\left(1 \vee|u|^{1 / \delta}\right)$ for all $u \in \mathbf{R}$. Then

$$
|(d L(a) \Phi)(g)| \leqslant\left(2 e^{b c^{2}} \tau^{-1} c_{0}+2^{-1} c_{2} \tau\right) G_{4^{-1} b, l}(g)
$$

for all $\tau \in\left\langle 0, t^{\delta / 2}\right]$ and $g \in G$. 
Proof: If $u \in \mathbf{R}$ then

$$
\left(|g|^{\prime}\right)^{2} \leqslant 2\left(|\exp (-u a) g|^{\prime}\right)^{2}+2\left(|\exp u a|^{\prime}\right)^{2} \leqslant 2\left(|\exp (-u a) g|^{\prime}\right)^{2}+2 c^{2}\left(1 \vee|u|^{2 / \delta}\right)
$$

and hence

$$
-\left(|\exp (-u a) g|^{\prime}\right)^{2} \leqslant-2^{-1}\left(|g|^{\prime}\right)^{2}+c^{2}\left(1 \vee|u|^{2 / \delta}\right)
$$

for all $g \in G$. Therefore

$$
|((I-L(\exp u a)) \Phi)(g)| \leqslant 2 e^{b c^{2}} c_{0} V^{\prime}(t)^{-1 / 2} e^{-2^{-1} b\left(|g|^{\prime}\right)^{2} t^{-1}}
$$

for all $g \in G$ and $u \in\left\langle 0, t^{\delta / 2}\right]$.

Using the identity

$$
f^{\prime}(0)=u^{-1}(f(u)-f(0))+u^{-1} \int_{0}^{u} d v(u-v) f^{\prime \prime}(v)
$$

with $f(u)=(L(\exp u a) \Phi)(g)$ one deduces that

$$
\begin{aligned}
|(d L(a) \Phi)(g)| & \leqslant u^{-1}|((I-L(\exp u a)) \Phi)(g)|+u^{-1} \int_{0}^{u} d v(u-v)\left\|d L(a)^{2} \Phi\right\|_{\infty} \\
& \leqslant 2 e^{b c^{2}} u^{-1} c_{0} V^{\prime}(t)^{-1 / 2} e^{-2^{-1} b\left(|g|^{\prime}\right)^{2} t^{-1}}+2^{-1} u c_{2} V^{\prime}(t)^{-1 / 2}
\end{aligned}
$$

for all $g \in G$ and $u \in\left\langle 0, t^{\delta / 2}\right]$. Then the lemma follows by setting $u=\tau e^{-4^{-1} b\left(|g|^{\prime}\right)^{2} t^{-1}}$. $\quad$

Next we prove the Gaussian bounds for the nilpotent derivatives of the kernel. If .$|\cdot|$ and $|\cdot|_{G_{N}}$ are moduli on $G$ and $G_{N}$ with respect to the basis $b_{1}, \ldots, b_{d}$, respectively, then it follows from (2) and the orthogonality of the $\bar{T}(g)$ that $|g|=|g|_{G_{N}}$ for all $g \in G_{N}$. Then by [15, Proposition III.42], for any neighbourhood $\Omega$ of the identity element there exists a $c>0$ such that

$$
c^{-1}|g|_{G_{N}} \leqslant|g|^{\prime} \leqslant c|g|_{G_{N}}
$$

or all $g \in G \backslash \Omega$.

Proposition 2.5. For all $\alpha \in J(\mathfrak{n})$ there are $b, c>0$ such that

$$
\left|B^{\alpha} K_{t}\right| \leqslant c t^{-\| \alpha \mid: / 2} G_{b, t}
$$

for all $t \geqslant 1$.

Proof: If $|\alpha|=0$ then the bounds (10) equal the bounds (1). The proof is by induction. Let $k \in\left\{d_{0}+1, \ldots, d_{q}\right\}, \alpha \in J(\mathfrak{n})$ and suppose that the bounds (10) are valid for $B^{\alpha} K_{t}$. Then by (9) and the inclusion " $\phi(R(t)) \subset B(c t)$ " in the proof of [15, Proposition IV.5.6], applied to the simply connected nilpotent group $Q_{N}$ it follows that there exists a $c>0$ such that $\left|\exp u b_{k}\right|^{\prime} \leqslant c|u|^{1 / \omega_{k}}$ for all $|u| \geqslant 1$. Then the bounds (10) follow for $B_{k} B^{\alpha} K_{t}$ from Lemma 2.4 by taking $\Phi=B^{\alpha} K_{t}, a=b_{k}, \delta=\omega_{k}$ and $\tau=t^{\omega_{k} / 2}$, using the induction hypothesis and the $L_{\infty}$-bounds of Corollary 2.3 on $B_{k}^{2} B^{\alpha} K_{t}$. Hence by induction the bounds (10) are valid for all $\alpha \in J(\mathfrak{n})$. The proof is complete. 


\section{Derivative bounds}

The derivative bounds on the kernel in a general direction follow from the derivative bounds in a nilpotent direction and an estimate on $\left\langle b_{j}, \operatorname{Ad}(g) b_{i}\right\rangle$. We need two lemmas before we prove the required bounds on $\operatorname{Ad}(g)$.

LEMMA 3.1. If $v \in \mathfrak{v}$ then $K(v) \mathfrak{q}_{N ; k} \subseteq \mathfrak{q}_{N ; k+1}$ for all $k \in\{1, \ldots, r\}$.

Proof: It is straightforward to prove that ad $v$ and $K(v)$ are derivations on $\boldsymbol{g}_{N}$. If $v^{\prime} \in \mathfrak{v}$ then $(\operatorname{ad} v) v^{\prime}=\left[v, v^{\prime}\right]_{N}$ and since $K(v)$ is a polynomial in ad $v$ without a constant term and ad $v$ is a derivation of $\mathfrak{q}_{N}$ it follows that $K(v) \mathfrak{v} \subseteq \mathfrak{q}_{N ; 2}$. Clearly $K(v) \mathfrak{n}=[v, \mathfrak{n}]_{N}$ $\subseteq \mathfrak{q}_{N ; 2}$. So $K(v) \mathfrak{q} \subseteq \mathfrak{q}_{N ; 2}$. Since $K(v)$ is a derivation of $\mathfrak{q}_{N}$ the corollary follows.

For all $\xi \in \mathbf{R}^{d_{q}}$ define $\|\xi\|=\sum_{i=1}^{d_{q}}\left|\xi_{i}\right|^{1 / w_{i}}$. Then it follows from the Campbell-BakerHausdorff formula and the first displayed formula on page 55 of [15] that there exists a $c>0$ such that

$$
\|\xi\| \leqslant c\left|\exp _{G_{N}} \xi_{1} b_{1} *_{G_{N}} \ldots *_{G_{N}} \exp _{G_{N}} \xi_{d_{q}} b_{d_{q}}\right|_{G_{N}}
$$

for all $\xi \in \mathbf{R}^{d_{q}}$ with $\|\xi\| \geqslant 1$.

Lemma 3.2. For all $i, j \in\{1, \ldots, d\}$ and $m_{1}, \ldots, m_{d_{q}} \in \mathrm{N}_{0}$ there exists a $c>0$ such that

$$
\begin{aligned}
\left|\left\langle b_{j},\left(\operatorname{ad} \xi_{d_{q}} b_{d_{q}}\right)^{m_{d_{q}}} \ldots\left(\operatorname{ad} \xi_{d_{0}+1} b_{d_{0}+1}\right)^{m_{d_{0}+1}} K\left(\xi_{d_{0}} b_{d_{0}}\right)^{m_{d_{0}}} \ldots K\left(\xi_{1} b_{1}\right)^{m_{1}} b_{i}\right\rangle\right| \\
\leqslant \begin{cases}c\|\xi\|^{\omega_{j}-\omega_{i}} & \text { if } \omega_{j} \geqslant \omega_{i} \\
0 & \text { otherwise }\end{cases}
\end{aligned}
$$

for all $\xi \in \mathbf{R}^{d_{q}}$ with $\|\xi\| \geqslant 1$.

Proof: By composition it suffices to prove the statement for $m_{1}+\ldots+m_{d_{q}}=1$. Let $l \in\left\{1, \ldots, d_{q}\right\}$ be such that $m_{l}=1$. Suppose the left hand side of (12) is not zero.

If $l \leqslant d_{0}$ then $v=\xi_{l} b_{l} \in \mathfrak{v}$ and one has $K(v) \mathfrak{m}=\{0\}$ and $K(v) \mathfrak{q}_{N ; k} \subseteq \mathfrak{q}_{N ; k+1}$ for all $k \in\{1, \ldots, r\}$ by Lemma 3.1. Hence $i \leqslant d_{q}$ and $\omega_{j}=w_{j}>w_{i} \geqslant \omega_{i}$. Therefore with $c=\left|\left\langle b_{j}, K\left(b_{l}\right) b_{i}\right\rangle\right|$ one has

$$
\left|\left\langle b_{j}, K\left(\xi_{l} b_{l}\right) b_{i}\right\rangle\right| \leqslant c\left|\xi_{l}\right| \leqslant c\|\xi\| \leqslant c\|\xi\|^{\omega_{j}-\omega_{i}}
$$

since $\|\xi\| \geqslant 1$.

If $l>d_{0}$ then with $n=\xi_{l} b_{l} \in \mathfrak{n}$ one has $(\operatorname{ad} n) b_{i}=-\left[b_{i}, n\right] \in \mathfrak{h}_{w_{l}} \cap \mathfrak{n}$ if $b_{i} \in \mathfrak{m}$. Hence $b_{j} \in \mathfrak{n}$ and $\omega_{j}-\omega_{i}=w_{j}=w_{l}$. Then $\left|\left\langle b_{j},\left(\operatorname{ad} \xi_{l} b_{l}\right) b_{i}\right\rangle\right| \leqslant\left|\left\langle b_{j},\left[b_{l}, b_{i}\right]\right\rangle\right|\|\xi\|^{\omega_{j}-\omega_{i}}$ since $\left|\xi_{i}\right| \leqslant\|\xi\|^{w_{l}}$. Alternatively, if $b_{i} \in \mathfrak{v}$ then $(\operatorname{ad} n) b_{i}=-\left(\operatorname{ad} b_{i}\right) n \in \mathfrak{q}_{N ; w_{l}} \cap \mathfrak{n}$. So $b_{j} \in \mathrm{n}$ and $\omega_{j}-\omega_{i}=w_{j} \geqslant w_{l}$. Then $\left|\left\langle b_{j},\left(\operatorname{ad} \xi_{l} b_{l}\right) b_{i}\right\rangle\right| \leqslant\left|\left\langle b_{j},\left[b_{l}, b_{i}\right]\right\rangle\right|\|\xi\|^{\omega_{j}-\omega_{i}}$ since $\left|\xi_{l}\right| \leqslant\|\xi\|^{w_{i}} \leqslant\|\xi\|^{w_{j}}$. Finally, if $b_{i} \in \mathfrak{n}$ then $(\operatorname{ad} n) b_{i} \in \mathfrak{q}_{N ; w_{i}+w_{l}}$. So $w_{j} \geqslant w_{i}+w_{l}$ and one can argue as before. 
LEMma 3.3. For all $i, j \in\{1, \ldots, d\}$ there exists a $c>0$ such that

$$
\left|\left\langle b_{j}, \operatorname{Ad}\left(g^{-1}\right) b_{i}\right\rangle\right| \leqslant \begin{cases}c\left(1+\left(|g|^{\prime}\right)^{\omega_{j}-\omega_{i}}\right) & \text { if } \omega_{j} \geqslant \omega_{i} \\ 0 & \text { otherwise }\end{cases}
$$

for all $g \in G$.

Proof: Since $G=M Q$ and $M$ is compact it follows that $\operatorname{Ad}(M) b_{i}$ is a bounded subset of $\mathfrak{m} \oplus \mathfrak{v}$ or $\mathfrak{h}_{w_{i}} \cap \mathfrak{n}$ if $b_{i} \in \mathfrak{m} \oplus \mathfrak{v}$ or $b_{i} \in \mathfrak{n}$, respectively. So it suffices to consider the case $g \in Q$. By [14, Theorem 3.18.11], there exists a $\xi \in \mathbf{R}^{d_{q}}$ such that $g=\exp \xi_{1} b_{1} \ldots \exp \xi_{d_{q}} b_{d_{q}}$. Then

$$
\operatorname{Ad}\left(g^{-1}\right)=e^{-\mathrm{ad} \xi_{d_{q}} b_{d_{q}}} \ldots e^{-\mathrm{ad} \xi_{d_{0}+1} b_{d_{0}+1}} e^{-K\left(\xi_{d_{0}} b_{d_{0}}\right)} \ldots e^{-K\left(\xi_{1} b_{1}\right)} e^{-S\left(\xi_{d_{0}} b_{d_{0}}\right)} \ldots e^{-S\left(\xi_{1} b_{1}\right)}
$$

But $S(v)$ is an orthogonal transformation, leaving $\mathfrak{m} \oplus \mathfrak{v}, \mathfrak{k}, \mathfrak{h}_{2}, \ldots, \mathfrak{h}_{\boldsymbol{r}}$ invariant. Moreover, $\exp _{G_{N}} \xi_{1} b_{1} *_{G_{N}} \ldots *_{G_{N}} \exp _{G_{N}} \xi_{d_{q}} b_{d_{q}}=\exp \xi_{1} b_{1} \ldots \exp \xi_{d_{q}} b_{d_{q}}=g$. Therefore by (11) and (9) it suffices to show that there exists a $c>0$ such that

$$
\left|\left\langle b_{j}, e^{-\mathrm{ad} \xi_{d_{q}} b_{d_{q}}} \ldots e^{-\mathrm{ad} \xi_{d_{0}+1} b_{d_{0}+1}} e^{-K\left(\xi_{d_{0}} b_{d_{0}}\right)} \ldots e^{-K\left(\xi_{1} b_{1}\right)} b_{i}\right\rangle\right| \leqslant \begin{cases}c\|\xi\| \|_{j}-\omega_{i} & \text { if } \omega_{j} \geqslant \omega_{i} \\ 0 & \text { otherwise }\end{cases}
$$

uniformly for all $\xi \in \mathbf{R}^{d_{q}}$ with $\|\xi\| \geqslant 1$. But this follows from Lemma 3.2 by expanding the (terminating) power series of the exponentials of the nilpotent endomorphisms.

Next we derive a global a priori Gaussian bound which will be improved by subsequent additional arguments.

LEMMA 3.4. For all $\alpha \in J(d)$ there exist $b, c>0$ such that

$$
\left|B^{\alpha} K_{t}\right| \leqslant c G_{b, t}
$$

for all $t \geqslant 1$.

PRoOF: Let $\alpha \in J(d)$. It follows from the semigroup property and the bounds (1) that there exists a $c>0$ such that

$$
\left\|B^{\alpha} S_{t}\right\|_{\infty}=\left\|B^{\alpha} S_{t}\right\|_{1 \rightarrow \infty} \leqslant\left\|B^{\alpha} S_{1 / 2}\right\|_{\infty \rightarrow \infty}\left\|S_{t-1 / 2}\right\|_{1 \rightarrow \infty} \leqslant c V^{\prime}(t-1 / 2)^{-1 / 2}
$$

for all $t \geqslant 1$. Hence there exists a $c>0$ such that $\left\|B^{\alpha} S_{t}\right\|_{\infty} \leqslant c V^{\prime}(t)^{-1 / 2}$ for all $t \geqslant 1$.

Now we prove the lemma by induction to $|\alpha|$. The bounds (13) are valid if $|\alpha|=0$. Let $k \in\{1, \ldots, d\}, \alpha \in J(d)$ and suppose the bounds (13) are valid for $B^{\alpha} K_{t}$. Then the bounds (13) for $B_{k} B^{\alpha} K_{t}$ follow from Lemma 2.4 by taking $\Phi=B^{\alpha} K_{t}, a=b_{k}, \tau=1$ and $\delta=1$, using the induction hypothesis and the above proved $L_{\infty}$-bounds on $B_{k}^{2} B^{\alpha} K_{t}$. Hence the lemma follows by induction.

The proof of Theorem 1.1 is an easy consequence of the next lemma. 
LEMMA 3.5. Let $R$ be a right invariant differential operator, $b, c>0, \delta \geqslant 0$ and $i \in\{1, \ldots, d\}$. Suppose that $\left|R K_{t}\right| \leqslant c t^{-\delta} G_{b, t}$ for all $t \geqslant 1$. Then there exist $b^{\prime}, c^{\prime}>0$ such that $\left|B_{i} R K_{t}\right| \leqslant c^{\prime} t^{-\delta} t^{-\omega_{i} / 2} G_{b^{\prime}, t}$ for all $t \geqslant 1$.

Proof: For all $j \in\{1, \ldots, d\}$ define $\psi_{i j}: G \rightarrow \mathbf{R}$ by

$$
\psi_{i j}(g)=\left\langle b_{j}, \operatorname{Ad}\left(g^{-1}\right) b_{i}\right\rangle .
$$

Then $B_{i} L_{G}(g)=\sum_{j=1}^{d} L_{G}(g) \psi_{i j}(g) B_{j}$ for all $g \in G$. Hence

$$
B_{i}(\psi * \varphi)=\sum_{j=1}^{d}\left(\psi \psi_{i j}\right) * B_{j} \varphi
$$

for all $i \in\{1, \ldots, d\}$ and $\varphi, \psi \in L_{1 ; \infty}(G)$. In particular,

$$
B_{i} R K_{2 t}=B_{i}\left(\left(R K_{t}\right) * K_{t}\right)=\sum_{j=1}^{d}\left(\psi_{i j} R K_{t}\right) * B_{j} K_{t}
$$

for all $t>0$. By Proposition 2.5 and Lemma 3.4 there exist $b_{1}, c_{1}>0$ such that $\left|B_{j} K_{t}\right|$ $\leqslant c_{1} t^{-\omega_{j} / 2} G_{b_{1}, t}$ for all $t \geqslant 1$ and $j \in\{1, \ldots, d\}$. Moreover, by Lemma 3.3, one can restrict the sum in (14) to $j \in\{1, \ldots, d\}$ with $\omega_{j} \geqslant \omega_{i}$. So suppose $\omega_{j} \geqslant \omega_{i}$. Then there exists a $c_{2}>0$ such that $\left|\psi_{i j}(g)\right| \leqslant c_{2}\left(1+\left(|g|^{\prime}\right)^{\omega_{j}-\omega_{i}}\right)$ for all $g \in G$. Therefore by the assumption on $R K_{t}$ one has

$$
\begin{aligned}
\left|\psi_{i j}(g)\left(R K_{t}\right)(g)\right| & \leqslant c c_{2}\left(1+\left(|g|^{\prime} t^{-1 / 2}\right)^{\omega_{j}-\omega_{i}} t^{\left(\omega_{j}-\omega_{i}\right) / 2}\right) t^{-\delta} G_{b, t}(g) \\
& \leqslant c c_{2}\left(1+c_{3}\right) t^{-\delta} t^{\left(\omega_{j}-\omega_{i}\right) / 2} G_{2^{-1} b, t}(g)
\end{aligned}
$$

for all $g \in G$ and $t \geqslant 1$, where $c_{3}=\sup _{x>0} x^{\omega_{j}-\omega_{i}} e^{-2^{-1} b x^{2}}$. Then

$$
\begin{aligned}
\left|\left(\psi_{i j} R K_{t}\right) * B_{j} K_{t}\right| & \leqslant c c_{1} c_{2}\left(1+c_{3}\right) t^{-\delta} t^{-\omega_{i} / 2} G_{2^{-1} b_{,} t} * G_{b_{1}, t} \\
& \leqslant c^{\prime} t^{-\delta} t^{-\omega_{i} / 2} G_{v^{\prime}, t}
\end{aligned}
$$

for some $b^{\prime}, c^{\prime}>0$, uniformly for all $t \geqslant 1$, since the convolution of two Gaussians is bounded by a Gaussian.

Proof of Theorem 1.1: The first estimate for $|\alpha|=0$ is given by (1) and it follows by induction to $|\alpha|$ from Lemma 3.5. The second estimate follows similarly once one can prove that $B_{i} K_{t}$ has bounds $\left|B_{i} K_{t}\right| \leqslant c t^{-1 / 2} G_{b, t}$ for all $t \geqslant 1$. Since $a_{1}, \ldots, a_{d^{\prime \prime}}$ is an algebraic basis it suffices to prove that for all $\alpha \in J\left(d^{\prime}\right)$ with $|\alpha| \geqslant 1$ there exist $b, c>0$ such that

$$
\left|A^{\alpha} K_{t}\right| \leqslant c t^{-1 / 2} G_{b, t}
$$

for all $t \geqslant 1$. The proof is again by induction. 
If $|\alpha|=1$ then (15) has been proved in [13, Proposition 1], for a sublaplacian and the proof for a complex subelliptic operator is the same, once one has the Gaussian bounds (1). Let $\alpha \in J\left(d^{\prime}\right)$ with $|\alpha| \geqslant 1, k \in\left\{1, \ldots, d^{\prime}\right\}$ and suppose the bounds (15) for $A^{\alpha} K_{t}$ are valid. Then the bounds (15) follow for $A_{k} A^{\alpha} K_{t}$ from Lemma 3.5 by writing $A_{k}$ as a linear combination of $B_{1}, \ldots, B_{d}$. Hence by induction the bounds (15) are valid for all $\alpha \in J\left(d^{\prime}\right)$ with $|\alpha| \geqslant 1$ and the proof of the theorem is complete.

\section{REFERENCES}

[1] G. Alexopoulos, 'An application of homogenization theory to harmonic analysis: Harnack inequalities and Riesz transforms on Lie groups of polynomial growth', Canad. J. Math. 44 (1992), 691-727.

[2] G. Alexopoulos, 'An application of homogenization theory to harmonic analysis on solvable Lie groups of polynomial growth', Pacific J. Math. 159 (1993), 19-45.

[3] N. Dungey, 'High order regularity for subelliptic operators on Lie groups of polynomial growth', (Research Report MRR02-006, The Australian National University, Canberra, Australia, 2002).

[4] N. Dungey, Private communication.

[5] A.F.M. ter Elst and D.W. Robinson, 'Subcoercive and subelliptic operators on Lie groups: variable coefficients', Publ. Res. Inst. Math. Sci. 29 (1993), 745-801.

[6] A.F.M. ter Elst and D.W. Robinson, 'Subelliptic operators on Lie groups: regularity', $J$. Austr. Math. Soc. Ser. A 57 (1994), 179-229.

[7] A.F.M. ter Elst and D.W. Robinson, 'Weighted subcoercive operators on Lie groups', $J$. Funct. Anal. 157 (1998), 88-163.

[8] A.F.M. ter Elst and D.W. Robinson, 'Second-order subelliptic operators on Lie groups III: Hölder continuous coefficients', Calc. Var. Partial Differential Equations 8 (1999), $327-363$.

[9] A.F.M. ter Elst and D.W. Robinson, 'Gaussian bounds for complex subelliptic operators on Lie groups of polynomial growth', Bull. Austral. Math. Soc. 67 (2003), 201-218.

[10] A.F.M. ter Elst, D.W. Robinson and A. Sikora, 'Heat kernels and Riesz transforms on nilpotent Lie groups', Coll. Math. 74 (1997), 191-218.

[11] A.F.M. ter Elst, D.W. Robinson and A. Sikora, 'Riesz transforms and Lie groups of polynomial growth', J. Funct. Anal. 162 (1999), 14-51.

[12] D.W. Robinson, Elliptic operators and Lie groups, Oxford Mathematical Monographs (Oxford University Press, New York, 1991).

[13] L. Saloff-Coste, 'Analyse sur les groupes de Lie à croissance polynômiale', Ark. Mat. 28 (1990), 315-331.

[14] V.S. Varadarajan, Lie groups, Lie algebras, and their representations, Graduate Texts in Mathematics 102 (Springer-Verlag, New York, 1984).

[15] N.T. Varopoulos, L. Saloff-Coste and T. Coulhon, Analysis and geometry on groups, Cambridge Tracts in Mathematics 100 (Cambridge University Press, Cambridge, 1992).

Centre for Mathematics and its Applications

Australian National University

Canberra, ACT 0200, Australia 